\title{
Effect of correlated noise on quasi-1D diffusion
}

\author{
D. V. Tkachenko ${ }^{1}$, V. R. Misko ${ }^{1}$, and F. M. Peeters ${ }^{1,2}$ \\ ${ }^{1}$ Department of Physics, University of Antwerpen, \\ Groenenborgerlaan 171, B-2020 Antwerpen, Belgium and \\ ${ }^{2}$ Departamento de Física, Universidade Federal do Ceará, 60455-900 Fortaleza, Ceará, Brazil
}

(Dated: October 31, 2018)

\begin{abstract}
Single-file diffusion (SFD) of an infinite one-dimensional chain of interacting particles has a longtime mean-square displacement $(\mathrm{MSD}) \propto t^{1 / 2}$, independent of the type of inter-particle repulsive interaction. This behavior is also observed in finite-size chains, although only for certain intervals of time $t$ depending on the chain length $L$, followed by the $\propto t$ for $t \rightarrow \infty$, as we demonstrate for a closed circular chain of diffusing interacting particles. Here we show that spatial correlation of noise slows down SFD and can result, depending on the amount of correlated noise, in either subdiffusive behavior $\propto t^{\alpha}$, where $0<\alpha<1 / 2$, or even in a total suppression of diffusion (in the limit $N \rightarrow \infty)$. Spatial correlation can explain the subdiffusive behavior in recent SFD experiments in circular channels.
\end{abstract}

PACS numbers: 05.40.-a, 66.10.C-, 83.10.Rs

First introduced in bio-physics [1] to account for the transport of ions through molecular-sized channels in membranes, the concept of single-file diffusion (SFD), i.e., diffusion of particles in a narrow (quasi-1D) channel where mutual passage is forbidden, has been extensively studied during the last few decades 2 -4] .

Recent advances in nanotechnology has stimulated a growing interest in SFD, in particular, in the study of transport in nanopores. Thus SFD was observed in experiments on diffusion of molecules in zeolite molecular sieves [5]. Zeolites with unconnected parallel channels serve as a realization of the theoretically investigated one-dimensional systems. Molecular diffusion of tetrafluoromethane in zeolite $\mathrm{AlPO}_{4}-5$ was studied by pulsed field gradient NMR spectroscopy [6]. The channel diameter was of the order of $0.73 \mathrm{~nm}$, whereas the diameter of the $\mathrm{CF}_{4}$ molecules was $0.47 \mathrm{~nm}$. Thus mutual passage of molecules was excluded and it was found that the movement of an isolated particle, after a short ballistic period, was determined by the stochastic interaction with the channel walls yielding diffusional behavior, and the MSD was shown to obey an anomalous diffusion law.

Despite the seemingly simplicity of SFD phenomenon, its theoretical description involves considerable difficulties caused by the necessity to solve the Fokker-Planck equation for the probability density distribution in a multiparticle system. Several studies tackled this problem for different model systems. Thus in Ref. 7] SFD of hardcore particles in a one-dimensional lattice was theoretically investigated by the method of "tagged vacancies" for an infinite chain, a finite chain with reflecting boundaries and chains with periodic boundary conditions. It was shown that for an infinite chain the mean-square displacement (MSD) of a diffusing particle depends on time as $\left\langle\Delta x^{2}\right\rangle \propto t^{1 / 2}$. The same result was obtained in Ref. [4] and, it was demonstrated in Ref. [8] that this result did not depend on the nature of the interparticle interaction. Note that this result was obtained assuming overdamped dynamics of diffusing particles while the problem remains unsolved for the general case. For the intermediate case of neither overdamped, nor underdamped dynamics numerical simulations were presented in Ref. [9].

In case of a finite chain with reflecting boundaries, the long-time MSD was shown [7] to become constant (i.e., independent of time $t$ ) which means a total suppression of diffusion. This result can be understood considering the fact that in a finite system (single-connected, nonperiodic and possessing no rotational degree of freedom), no matter how large, only a finite set of configurations of particles can be realized. Thus for a long enough observation time, the system accesses all the states in configuration space. An exact analytical proof of this result using the Bethe-ansatz was recently presented in Ref. [10]. A number of numerical studies on the SFD phenomenon have been performed using Langevin dynamics (see, e.g., Ref. 11]) including SFD on periodic substrates [12 14.

It is hard to fulfill the SF condition and investigate SFD experimentally for atomic systems. Alternatively, experimentalists used micro-meter sized colloidal particles in narrow channels. A well-defined SFD model was created [15] by confining paramagnetic colloidal spheres of $3.6 \mu \mathrm{m}$ in a set of circular trenches $(7 \mu \mathrm{m}$ in width and 33 to $1608 \mu \mathrm{m}$ in diameter) fabricated by photolithography. Using video microscopy, the trajectories of individual particles were followed over long periods of time and revealed a SFD behavior characterized by $\mathrm{MSD} \propto t^{1 / 2}$.

SFD was recently realized even on a macroscopic scale. The advantage of using a macroscopic system is that it allows one to easily fulfill the SF condition and observe the motion of particles using simple tools such as an optical microscope. The diffusion of such macroscopic charged metallic balls (of radius $R=0.4 \mathrm{~mm}$ and mass $m=2.15$ $\mathrm{mg}$ ) was investigated in Ref. [16]. The balls were interacting electrostatically and moving in a circular channel such that mutual passing was forbidden, while mechanical shaking induced an effective temperature. The authors found that the system of interacting balls exhibited a subdiffusive behavior $\propto t^{1 / 4}$, i.e., slower than that pre- 
dicted for SFD and observed in colloidal systems.

Here we investigate the effect of correlations of noise that causes diffusion. In particular, we study SFD of a finite-size system (i.e., interacting particles diffusing in a closed circular channel) driven by a mixture of a totally uncorrelated noise and a spatially-correlated (although uncorrelated in time) noise. Being coordinateindependent, spatially-correlated noise can be also referred to as "global". We show that the presence of a spatially-correlated component dramatically changes the SFD behavior, and it can also be used as an alternative explanation of the slowing down of diffusion observed in experiments with circular channels driven by artificial stochastic noise modeling temperature.

The model. - Let us consider SFD of interacting particles in a circular channel driven, by non-correlated and spatially-correlated stochastic forces. The fact that both forces are stochastic implies the absence of time correlations between the forces of the same component as well as of mutual correlations between different components. The fraction of the correlated noise to the total noise is given by a parameter $\lambda$, where $0 \leq \lambda \leq 1$. Therefore the fraction of non-correlated noise is $1-\lambda$. The motion for the $i$-th particle is described by the Langevin equation,

$$
\begin{aligned}
m \frac{d^{2} \vec{r}_{i}}{d t^{2}} & =-\gamma \frac{d \vec{r}_{i}}{d t}-\sum_{j, i \neq j} \nabla \vec{U}\left(r_{i j}\right)-\nabla \vec{U}_{c o n f}\left(r_{i}\right) \\
& +\lambda \vec{F}_{c}+(1-\lambda) \vec{F}_{n c, i}
\end{aligned}
$$

where $r_{i}$ is the radius of $i$-th particle, $m$ is its mass, $\gamma$ is the friction coefficient. The non-correlated part of noise $\vec{F}_{n c, i}=\left\{F_{n c, i}^{x}(t), F_{n c, i}^{y}(t)\right\}$ and the spatially-correlated part $\vec{F}_{c}=\left\{F_{c}^{x}(t), F_{c}^{y}(t)\right\}$ obey the following conditions,

$$
\begin{aligned}
& \left\langle F_{n c, i}^{k}\left(t^{\prime}\right)\right\rangle=0,\left\langle F_{c}^{k}\left(t^{\prime}\right)\right\rangle=0, \\
& \left\langle F_{n c, i}^{k}\left(t^{\prime}\right) F_{n c, j}^{l}\left(t^{\prime \prime}\right)\right\rangle=2 \gamma k_{B} T \delta_{i, j} \delta_{k, l} \delta\left(t^{\prime}-t^{\prime \prime}\right), \\
& \left\langle F_{c}^{k}\left(t^{\prime}\right) F_{c}^{l}\left(t^{\prime \prime}\right)\right\rangle=2 \gamma k T \delta_{k, l} \delta\left(t^{\prime}-t^{\prime \prime}\right), \\
& \left\langle F_{c}^{k}\left(t^{\prime}\right) F_{n c, i}^{l}\left(t^{\prime \prime}\right)\right\rangle=0,
\end{aligned}
$$

where $k, l=x, y$ are the coordinate indices and $i, j$ are the particle indices. Such a choice of the correlation relations (2) ensures that the system eventually approaches an equilibrium state with temperature $T$ at any time [12]. The interparticle interaction potential $U\left(r_{i j}\right)$ in our calculations was taken a Yukawa potential, $U(r)=\frac{q^{2}}{\varepsilon_{0}} \frac{e^{-\kappa r}}{r}$. Here $\kappa=1 / \Lambda$ where $\Lambda=4.8 \cdot 10^{-4} m$ [16]. In our numerical calculations, other parameters of the system were also taken from the experiment [16]. The general conclusions are independent of the exact functional form of the interaction potential. The confinement potential, $U_{\text {conf }}\left(r_{i}\right)$, which restricts the radial motion of the particles within the circular channel is taken parabolic: $U_{\text {conf }}\left(r_{i}\right)=\beta\left(r_{c h}-r_{i}\right)^{2}$, where $\beta$ is chosen from the condition $V_{g s}=\beta r_{0}^{2}$, where $r_{0}$ is the radius of the particle and $V_{g s}$ is the ground state energy, i.e., the energy of the configuration when all the particles are distributed equidistant near the circular channel "bottom".
Long-time SFD in a finite-size chain. - In Ref. [7], SFD of noninteracting (i.e., hard-core) particles diffusing in a ring was studied theoretically using the "tagged particle" method. It was shown that the long-time MSD grew as $2 D t$, where the diffusion coefficient $D$ was inversely proportional to the density of particles.

We will demonstrate that this result is also justified for a closed (e.g., circular) chain of interacting particles. For this purpose, let us first notice that SFD in a finite system has two characteristic lengths: (i) the average interparticle distance, $a$, and, as distinct from infinite systems, (ii) the length of the chain, $L$. The corresponding hierarchy of times raising from the two characteristic lengths, implies that along with the "long-time" regime when a particle diffuses for a distance of the order of the average interparticle distance $a$ (during time $t_{a}$ ), there is another time scale, $t_{L}$, which determines the long-time behavior. This is the time required for a particle to diffuse over a distance comparable to the system length, $L$. Note that for large systems with $N \gg 1$ particles, $t_{L} \gg t_{a}$ (and $t_{L} \rightarrow \infty$ when $N \rightarrow \infty$ ). It is clear that due to the single-filing condition, diffusion of all particles will be approximately the same for $t \geq t_{L}$ and thus can be described by a "collective" coordinate $\vec{R}=(1 / N) \sum_{i} \vec{r}_{i}$.

In case of a circular chain, one can define this collective coordinate as an average angle of "rotation" of the system as a whole, $\phi=\left\langle\phi_{i}\right\rangle=(1 / N) \sum_{i} \phi_{i}$. The angular diffusion of $i$ th particle is described by the system of equations which follow from Eqs. (11),

$$
\begin{aligned}
J_{i} \frac{d^{2} \phi_{i}}{d t^{2}} & =-\gamma^{\prime} \frac{d \vec{\phi}_{i}}{d t}-\sum_{j, i \neq j} \vec{r}_{i} \times \nabla \vec{U}\left(r_{i j}\right) \\
& +\lambda \vec{r}_{i} \times \vec{F}_{c}+(1-\lambda) \vec{r}_{i} \times \vec{F}_{n c, i}
\end{aligned}
$$

where $J_{i}$ is the moment of inertia of $i$-th particle, and $\gamma^{\prime}=\gamma r_{c h}^{2}$. Taking the average over all the particles, we have:

$$
\begin{aligned}
\frac{1}{N_{p}} \sum_{i} J_{i} \ddot{\phi}_{i} & \simeq J \ddot{\phi}=-\gamma^{\prime} \dot{\phi}+M_{\text {inter }} \\
& +\lambda M_{c}+(1-\lambda) M_{n c}
\end{aligned}
$$

where we assume $J \simeq J_{i}$, i.e., neglect the radial dispersion, and

$$
\begin{aligned}
M_{\text {inter }}= & -\frac{1}{N_{p}} \sum_{i} \sum_{j, i \neq j} \vec{r}_{i} \times \vec{\nabla} U\left(r_{i j}\right)=0 \\
M_{c} & =\frac{1}{N_{p}} \sum_{i} \vec{r}_{i} \times \vec{F}_{c} \\
& \simeq \oint_{\text {channel }} d \phi \vec{r}_{i} \times \vec{F}_{c}=0 \\
M_{n c} & =\frac{1}{N_{p}} \sum_{i} \vec{r}_{i} \times \vec{F}_{n c} .
\end{aligned}
$$


It is worth noting that expression (5) is exact due to the sine theorem. This well-known result from the dynamics of a conservative system is applicable to the "conservative part" of our system (although the whole system is not conservative). Relation (6) means that the field of stochastic spatially-correlated force is homogeneous in space, so the integral of this force along any closed contour is equal to zero. In other words, the action of the spatially-correlated force on half of particles in the channel is on average compensated by its action on the other half of particles. Thus the equation of motion for the average angle $\phi$ that characterizes the collective rotation of the system is:

$$
J \ddot{\phi}=-\gamma \dot{\phi}+(1-\lambda) M_{n c} .
$$

This equation is isomorphic to the equation of motion of a free particle driven by a stochastic force. As a consequence, the long-time SFD behavior of a free particle can be projected on the angular diffusion described by Eq. (8). Integrating Eq. (8) we obtain the expression for the long-time angular MSD: $\left\langle\Delta \phi^{2}\right\rangle=2 D_{\phi} t$. Thus we can conclude that long-time SFD (i.e., when $t \geq t_{L}$ ) related to the collective rotation of the system of particles in a circular channel is determined only by the stochastic spatially-non-correlated force. In other words, the interparticle interaction and stochastic spatially-correlated force do not influence long-time diffusion. On the other hand, if the system is driven only by spatially-correlated noise $(\lambda=1)$, the stochastic term in Eq. (8) is zero, thus resulting in the total suppression of long-time SFD.

We stress that these preliminary conclusions are based on Eqs. (3)-(8) for "collective" coordinate $\phi$ which are justified in the limit when $t \rightarrow \infty$ and $N \rightarrow \infty$ and can be used only for a qualitative prediction of the diffusive behavior in large $(N \gg 1)$ finite systems in the longtime limit $t \geq t_{L}$. Our exact results presented below are obtained by numerically integrating Eqs. (10).

The results of numerical calculations of the MSD of the SFD of $N$ particles in a circular channel are shown in Fig. 1 for two limiting cases: (i) for $N=20, \lambda=0$, i.e., for purely non-correlated noise (Fig. 1(a)), and (ii) for $N=20,30$, and $45, \lambda=1$, i.e., for fully spatiallycorrelated noise (Fig. 1(b)). In case $\lambda=0$, the initial fast grow (i.e., $\propto t^{2}$ followed by $\propto t^{1}$ ) of the MSD is followed by the pronounced $\propto t^{1 / 2}$ "long-time" (for infinite systems) region (for $t_{a} \leq t \leq t_{L}$ ) gradually changing to $\propto t^{1}$ asymptotic behavior for $t>t_{L}$ (Fig. 1(a)) which is common for any finite chain and any $\lambda$. The short-time behavior for $\lambda=1$ is rather similar, while for longer time the $t^{1}$ growth rapidly changes to a subdiffusive behavior $\propto t^{\alpha}$, with $0<\alpha<1 / 2$, gradually approaching a $t^{0}$-plateau for large $N$ [17] (see Fig. 1(b)), in agreement with Eq. (8).

Spatially-correlated vs. non-correlated noise. - In our numerical study of angular diffusion in a circular channel the standard deviation of an average angle (i.e., the
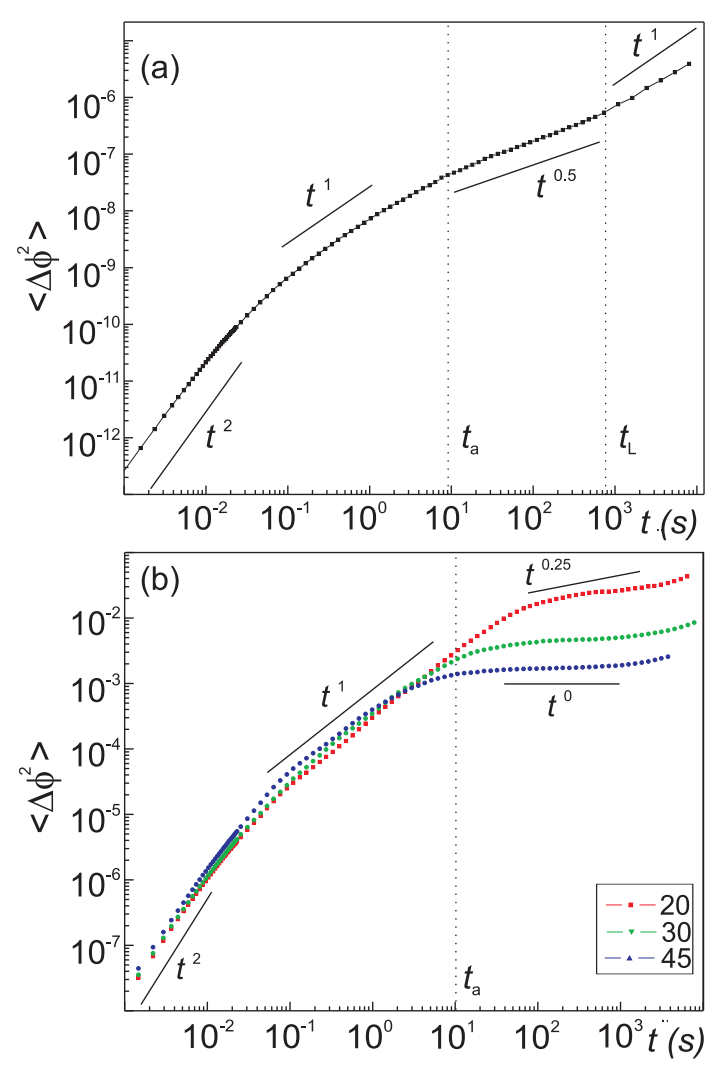

FIG. 1: (Color online) The average MSD (in log-log scale) of a particle diffusing in a circular channel with $N$ particles, driven by: (a) purely stochastic noise $(\lambda=0)$, for $N=20$, and (b) fully spatially-correlated noise $(\lambda=1)$, for $N=20$, 30, and 45. $\phi$ is measured in radians (also in Fig. 2). For $\lambda=0$, the initial fast grow $\propto t^{2}$ and $\propto t^{1}$ of the MSD is followed by the $\propto t^{1 / 2}$-region (for $t_{a} \leq t \leq t_{L}$ ) which gradually evolves to the long time $\left(t>t_{L}\right) \propto t^{1}$ asymptotic behavior (a). For $\lambda=1$ (b), the $t^{1}$ growth rapidly changes by a subdiffusive behavior $\propto t^{\alpha}$, with $0<\alpha<1 / 2$.

MSD),

$$
\left\langle\left\langle\Delta \phi^{2}\right\rangle_{e}\right\rangle_{p}=\frac{1}{N_{p} N_{e}} \sum_{i, j}\left(\left\langle\Delta \phi_{i, j}^{2}\right\rangle_{t}-\left\langle\Delta \phi_{i, j}\right\rangle_{t}^{2}\right)
$$

was calculated as a function of time, $t$. Here $\langle\ldots\rangle_{p}$ denotes averaging over all particles of the given ensemble, $\langle\ldots\rangle_{e}$ over various ensembles, and $\langle\ldots\rangle_{t}$ over time. The number of ensembles was chosen typically from 100 to 300, for $N=5$ to 45 particles in the ensemble.

Results of simulations for different $\lambda$ show (Fig. 2(a)) that when the fraction of the correlated noise increases, the region of fast growth (similar to the behavior of a free particle) of SFD $\propto t$ expands, while the region of subdiffusive growth, i.e., $\propto t^{1 / 2}$ (typical for SFD of a particle in an infinite chain), is essentially narrowed and rapidly changes to even stronger subdiffusive mode $\propto t^{\alpha}$, with $0<\alpha<1 / 2$. Thus we observe a considerable slowing down of diffusion on a long-time scale when increasing the fraction of spatially-correlated noise. Note that for some value of $\lambda$, i.e., $\lambda \approx 0.6$, the long-time $\alpha$ becomes 

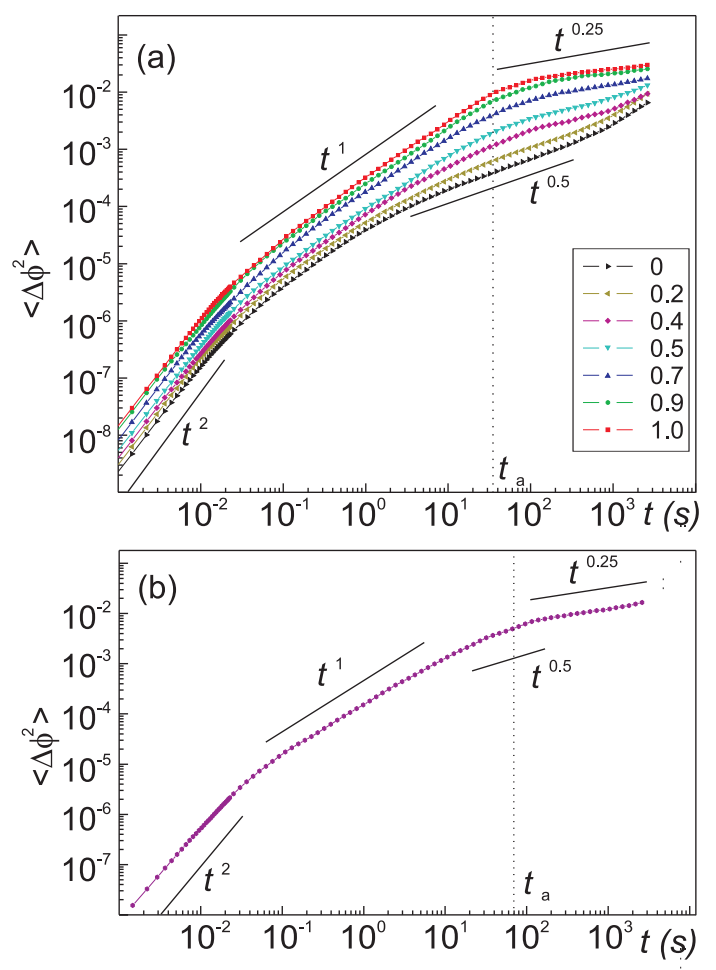

FIG. 2: (Color online) The average MSD (in log-log scale) of a particle, driven by a mixture of non-correlated and spatiallycorrelated noise, for: $\lambda=0,0.2,0.4,0.5,0.7,0.9$, and 1 (a). With increasing $\lambda$, the region of fast growth $\propto t$ expands, while the region of subdiffusive growth, i.e., $\propto t^{1 / 2}$, shrinks and rapidly changes to a stronger subdiffusive mode $\propto t^{\alpha}$, with $0<\alpha<1 / 2$, including $\propto t^{1 / 4}$ for $\lambda=0.6$ (b).

$\approx 0.25$, i.e., leading to a pronounced region in the MSD $\propto t^{1 / 4}$ (Fig. 2(b)). In the limit of completely spatiallycorrelated noise $(\lambda=1)$, the calculated $\operatorname{MSD}\left\langle\Delta \phi^{2}\right\rangle$ saturates for large time $t$ (for sufficiently large number of particles, see Fig. 1(b)).

The observed saturation of the MSD for long-time scale looks similar to that found in a finite chain where the motion of particles was restricted in the direction of the chain that in turn was related to the fact that for long time (of the order of the time of diffusion of a particle over the whole system) the trajectory of the particle filled practically all the configurational space accessible to the given particle. However, in spite of the similarity in the long-time SFD behavior with, e.g., a finite chain of particles in the presence of reflecting boundaries [10], the physics behind the observed slowing down of the diffusion in our system is completely different. Our system is periodic and it possesses a rotational degree of freedom which leads to a single-particle-like long-time asymptotic
SFD behavior (as shown in Fig. 1(a)). Adding a fraction of spatially-correlated noise effectively suppresses the rotational degree of freedom and thus the SFD in the longtime limit.

Our results provide an alternative explanation of the subdiffusive behavior (i.e., slower than $\propto t^{1 / 2}$ ) of particles diffusing in a circular channel driven by an $a r$ tificial stochastic force. For example, in SFD experiments with charged balls [16], the system temperature was modeled by random acoustic waves produced by a set of loudspeakers placed under the substrate with a circular channel. Thus the whole sample was shaked and, consequently, different particles were not driven individually. The spatial correlation of noise (although being uncorrelated in time) in this system arises, in our opinion, due to two factors: (i) the collective excitation of all the particles in the system by a limited set of sources of noise (note that even a large number of different sources would lead to spatial correlation of noise, due to the interference between the waves), and (ii) shaking the whole sample containing the channel with all the particles embedded in a single-file chain. Note that as was suggested by the authors of Ref. [16] and confirmed by recent numerical simulations [9], the interparticle interaction in a non-overdamped regime perhaps could also lead to a slowing down of the long-time SFD in circular channels.

Similar conditions, leading to spatially-correlated noise, can also be realized in other artificial systems that exhibit single-file behavior.

Conclusion. - We have investigated the effect of spatial correlation in noise on single-file diffusion of interacting particles diffusing in a finite-size circular channel where the long-time diffusion is characterized by two time regimes, $t_{a}<t<t_{L}$ and $t>t_{L}$. We demonstrated that in the limit of purely non-correlated noise, the mean-square displacement characterizing the diffusion rate shows a $\propto t^{1 / 2}$ (for $t_{a}<t<t_{L}$ ) and a $\propto t^{1}$ (for $t>t_{L}$ ) behavior independent of the type of the interparticle interaction. Adding a fraction of spatiallycorrelated noise slows down the diffusion and could result in either subdiffusive behavior $\propto t^{\alpha}$ with $\alpha<1 / 2$ (for $t_{a}<t<t_{L}$ ), or even in the total suppression of diffusion on a long-time scale (for $N \rightarrow \infty$ ). Our model provides an alternative explanation of experimentally observed slowing down of the diffusion rate of interacting particles driven by artificial noise sources.

We acknowledge discussions with M. Saint-Jean. This work was supported by the "Odysseus" program of the Flemish Government and FWO-Vl.
[1] A. L. Hodgkin and R. D. Keynes, J. Physiol. 128, 61 (1955).

[2] David G. Levitt, Phys. Rev. A 8, 3050 (1973).
[3] Peter A. Fedders, Phys. Rev. B 17, 41 (1978).

[4] Jörg Kärger, Phys. Rev. A 45, 4173 (1992).

[5] W. M. Meier and D. H. Olsen (Eds.), Atlas of Zeo- 
lite Structure Types, Butterworths-Heinemann, London (1992).

[6] K. Hahn, J. Kärger, and V. Kukla, Phys. Rev. Lett. 76, 2762 (1996).

[7] H. van Beijeren, K. W. Kehr, and R. Kutner, Phys. Rev. B 28, 5711 (1983).

[8] M. Kollmann, Phys. Rev. Lett. 90, 180602 (2003).

[9] K. Nelissen, V. R. Misko, and F. M. Peeters, Europhys. Lett. 80, 56004 (2007).

[10] L. Lizana and T. Ambjörnsson, Phys. Rev. Lett. 100 200601 (2008).

[11] A. Taloni and M. A. Lomholt, Phys. Rev. E 78, 051116 (2008).
[12] A. Taloni and F. Marchesoni, Phys. Rev. Lett. 96020601 (2006).

[13] S. Savel'ev et al., Phys. Rev. E 74, 021119 (2006).

[14] S. Herrera-Velarde and R. Castañeda-Priego, Phys. Rev. E 77, 041407 (2008).

[15] Q.-H. Wei, C. Bechinger, and P. Leiderer, Science 287, 625 (2000).

[16] G. Coupier, M. Saint Jean, and C. Guthmann, Phys. Rev. E 73, 031112 (2006).

[17] Note that the $t^{0}$ regime is achieved only for $N \rightarrow \infty$ when $t_{L} \rightarrow \infty$ and thus the long-time regime $t \geq t_{L}$ cannot be reached. 